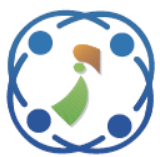

\title{
Secure Decision-Making Approach to Improve Knowledge Management Based on Online Samples
}

\author{
Kannan Kaliyan $^{1 *} \quad$ Raja Kothandaraman ${ }^{2}$ \\ ${ }^{1}$ Faculty of Computer Science and Engineering, Sathyabama University, Chennai, India \\ ${ }^{2}$ Dhaanish Ahmed College of Engineering, Chennai, India \\ * Corresponding author's Email: kannankphd@gmail.com
}

\begin{abstract}
Data is bombarding in every area of the industry and information gathered from the available data is the raw material for the online society. By the evolution of the telecom sector and various user-preferred applications, the internet has become inevitable. It, one of the huge technologies that are going to dominate the internet society, is the much expected technological evolution of this decade. Since everything is made online, all the activity happening \& happened should be stored/retrieved in an efficient way. In this paper, we propose a decision process that has decision-making process and game theory along with a coding technique to acquire confident knowledge from the available data set in a secure way. Our mechanism is simulated and the results show a considerable improvement in the decision-making process. Also, analysis proves our algorithm is better in terms of quality parameters than the existing algorithms.
\end{abstract}

Keywords: Decision support system, Executive information systems, Knowledge management, Decision making with game theory, Online samples.

\section{Introduction}

Decision support systems help in decisionmaking by assembling helpful data from a mix of raw information, records, and person's knowledge. In1980s, Single user models of Decision Support System, Organizational Decision Support Systems (ODSS), Group Decision Support Systems (GDSS) and Executive Information Systems (EIS). In 1990 DSS extended to the greater level of data analysis, which included the techniques of data warehousing and online analytical processing. DSS also used to identify a specified problem and helps to solve it. The nature of a DSS may be fully computerized, human-powered or a combination of both. An interactive software-based system tends to be a properly designed DSS. The framework of the system consists of four phases. An intelligence phase of conditional search which calls a decision, Design phase to analyze and develop various alternatives actions of the solution, action selection from the various cases is done by choice phase and finally for adopting the selected action on the decision condition. Interrelated, Personal Support and Organizational Support are the three distinct categories of Decision Support System. The components of running a DSS successively are a set of inputs which may contain factors or numbers and characteristics to analyze, a manual analysis of user knowledge and expertise, data transformed into decisions as outputs and the results generated based on user criteria from the set of decisions. A large amount of data can be easily understood by means of a DSS. A great advantage of DSS is that can be programmed to generate various types of reports based on user specifications and can also represent the given data graphically in the form of graphs. DSS applications are in various fields such as medical diagnosis, customer verification process in banks, E-Governance management, farm and policy level agricultural management, evaluation of defective rails in railroads, etc. The limitations that arise in these systems are complex in quantifying the data, assumption unawareness, information overload 
leaves in a dilemma to consider which data and not to consider which data.

To analyze the scientific models of contention and collaboration between the intelligent decision makers Game hypothesis was proposed. Game theory was developed in 1950's and the theory was applied to the biological field later. The prisoner's dilemma was the first mathematical discussion of the Game theory around a similar time John Nash developed the Nash equilibrium proving that every n-player, non-zero-sum which resulted in noncooperation game. The Game types in Game theory was cooperative and non-cooperative, symmetric and asymmetric, zero-sum and non-zero-sum, synchronous and consecutive. In a Cooperative game, the players are allowed to bind to each other through laws, whereas in a non-cooperative game, in the event that players can't shape together or the understandings should act naturally implemented by means of credible threats. The settlements for playing a specific procedure depend just on the methodologies utilized and not on the player in a symmetric game. Detainee's situation and the stag chase are techniques for the symmetric game. The asymmetric game provides the strategy for the players to be non-identical. The Ultimatum game and the dictator game are examples of asymmetric games. An exceptional instance of constant-sum games are the Zero-sum games through which the players can increment or lessening the resources that are available. If the outcome of a game is greater than or less than zero, it is given as a non-zero-sum game. A special case of non-zero-sum recreations is that a pick up by one player does not really compare with a misfortune by another player. Poker exemplifies the zero-sum game, though detainee's quandary represents the non-zero-sum game. In a simultaneous game both the player is allowed to play at the same time and in a sequential game, the next player will have some knowledge about the actions done by the earlier player. Decision trees are an example of sequential while payoff matrices are a great example of the simultaneous game. Extensive form, Normal form, and characteristic function form are the representation types of a game by means of game theory. The games that are played in sequencing moves are represented by means of a tree, the normal form of a game is represented by means of a matrix usually and game that contains separate rewards, removable utility, and the decision of payoff of each unity respectively. The game has been utilized to examine a wide assortment of practices by human and animals. In the field of political science, it is used to classify the special interest groups and politicians. For equating fitness through playoff games in the biological sector. Interactive epistemology, the means of a group to have normal convictions or knowledge in philosophy. In the field of computer science and logic, several algorithms have been proposed for modeling the game semantics and interactive computations. For demonstrating lower limits on the computational intricacy of randomized algorithms Yao's principle is used. The main drawbacks of the Game theory are solving games with mixed strategies, including a large payoff matrix is very complex, a different equilibrium will be derived if a different assumption chooses, for a non-zero sum game mathematical solutions are always not possible.

For an effective data analysis, the steps of creating, sharing and managing the knowledge gathered in an organization are very important, this process is called as Knowledge Management (KM). Knowledge should be used at its best to achieve objective effectively and efficiently. Knowledge Management focuses on improving performance, innovative ideas, sharing of ideas learned, integration factors and improvement factors. In 1990s KM was supported by individual practitioners to make their individual organizations benefited, then gradually moved towards academic maturity. The core components of Knowledge Management include people and their culture, processes and their structures and the technology implemented. Three frameworks were designed for distinguishing different types of knowledge that exist in a system. First framework that categorizes the knowledge into two tacit knowledge and explicit knowledge. Tacit Knowledge speaks to the client's knowledge that the user is aware of. The second one is for categorizing the knowledge dimensions that differentiates knowledge of a system of a human individual. The third framework distinguishes the new knowledge and the already established knowledge in a social environment. The strategy for knowledge management is classified into three categories. The user is allowed to feed newly gained knowledge into a repository of shared knowledge provided by other users is called as push strategy or actively managing knowledge. In second criteria the user requests for sharing of knowledge that is already provided by experts on an ad hoc basis or pull strategy. A third strategy is called personalization in which the individuals share their knowledge directly. Knowledge Management contains the following steps in a cycle, the creation of knowledge, Sharing of the created knowledge, Structuring of the knowledge, using the provided knowledge and auditing the knowledge and recreating it. As there 
are so many merits there, are also demerits in the system sharing and information and knowledge may not suitable for every environment. The implementation of these systems is costly and complex to understand. The timing of the crisis will expect the next form of knowledge. In this paper, we propose a game theory based knowledge management solution along with coding techniques that considerably predicts the decision that is required to take a decision in an uncertain environment. A coding technique helps in making the data transmission in a secure and reliable way.

This paper is organized as follows. Section 2 deals with the related works involved in the knowledge management, game theory, and decisionmaking. Section 3 elaborates the proposed model and Section 4 describes the performance analysis of our proposed mechanism in various scenarios. Section 5 concludes the article with the future scope of the enhancements.

\section{Related works}

Daniel McDuff, et al., [1] created a data set containing facial reactions of the users by an implementation of computer vision algorithm for code sign and message judgment that relies on the facial muscle movements. A video data is collected by recording the responses in everyday environments via the viewer's web camera. This online data set collected is used for calculating reliable normal distributions across various media. When it comes to large-scale database facial behaviours will be difficult for interpreting with another factor measured data set. Ren'eGrzeszicket, et al., [2] used the bag of features approach through the low computational cost of online processing is achieved. The possibility of fusing the results of multiple channels to improve its robustness. DCASE 2013 is live office dataset and ITC-IRST datasets are used. Huijie Lin et al., [3] experimented stress detection based on the data sets from the real world social platform through the messages from and to the friends of the user. Based on this proposed a combination of factor graph model and a novel hybrid model with Convolution neural network to leverage the tweet content. This model gave an increase of 6-9 percent in F1 scores. Lequan $\mathrm{Yu}$ [4] proposed a novel disconnected and online profound learning reconciliation for detecting colorectal cancer. It implemented a $3 \mathrm{~d}$ framework which developed an integration method to overcome the variations in the polyps feature attributes. MICCAI 2015 was the data set that was used here. Thomas Zhang, et al., provides line classification for health systems to give relevant information to users accordingly. Implementation of Multiple Support Vector Machine (SVM) classified 1192 data sets which resulted in a maximum prediction of $75 \%$. Classification performance was considerably lower in this method [5]. A collection of multifaceted motion segmentation benchmarks containing the trajectory and ground-based truth. The data sets included a set of real life and short sequences with the increased number of motions and frames per sequence proposed by Muhammad Habib Mahmood, et al., [6]. Cynthia Grajedaet, et al., [7] provided an overview of the datasets that are available. Describes the origin of the data set with the comparison of the real world and synthetic data sets, the data sets that are released by the researchers and the types of data set that exist to provide full information on the data sets that are available for the digital forensic implications. Helen Caple and John S. Knox [8] combined the pictures on large data sets to provide new information from the created picture. The data sets were taken from the 2009 and 2013 emergence of new genres gallery. A potentially emerging genre from identification of two genres and combining them. Jing Zhang, et al., [9] used RGB-D (Red, Green, Blue, and Depth) action based data sets to provide action recognition of human. This provided detailed analysis and information guiding insightful resource by selecting the data sets which resulted in a protocol for selection and evaluation of data sets.

Hemant Jalotaet, et al., [10] proposed a Decision Support System for generation of a suitable portfolio for an investor in an uncertain environment through a multi-criteria framework. Return and liquidity of various attributes using L-R fuzzy numbers. An Entropy-Cross-Entropy (ECE) algorithm which provides a method for finding the solution for optimization problem which is meant for finding the best fit L-R fuzzy number with respect to the corresponding indeterminate parameters. Once the L-R fuzzy rule is generated the portfolio optimization problem is solved by Hybrid Intelligence Algorithm (HIA) which is developed by embedding fuzzy simulation inside "MIBEX-SM" genetic algorithm. Hong chi et al., [11] built up a Decision Support System for detecting crimes with a feature similarity algorithm which calculates the pairwise similarity and in building a classifier decides whether a case pair belongs to a series or not. The system also has flexibility in training data update and domain expert interaction which includes the adjustment of similarity matrices and decision thresholds which act as the key components. These factors lead to a good trade-off between caseload 
and true link pairs. This approach cannot directly deal with the textual content which is a major drawback. Ana Raquel Xambreet, et al., [12] tested a Decision Support System for a superior plan of Cellular Manufacturing Systems (CMS). Through this resource are grouped to create independent cells capable of complete processing similar parts of the cellular system. The decision is given with the help of solid data and sustained models inside the database. A Flexible Irrigation Scheduling Decision Support System (FIS-DSS) was proposed by Gaiqiang Yang, et al., [13] keeping in mind the end goal to allocate suitable water allocation schemes. A fuzzy interim programming model with different targets and conditions with points of interest in information handling, an adaptable model, the other explaining algorithm and an outcome show. The inference engine uses the $\mathrm{PC}$ projects to get the learning base; the information base is utilized to give all the data and principles for the inference engine. A multi-agent decision support system, ideology was proposed by StanisawaKluskaNawareckaet, et al., [14]. This technology solved the decentralized problem and creating complex decision support systems, simulation, reasoning and machine learning which gave a distributed knowledge. Decomposition of an inference algorithm into separated modules and distribution of knowledge base into several parts is done. This was suitable for a system that can work as a platform for integration of heterogeneous knowledge sources. BhornswanThanathornwonget, et al., [15] developed a clinical decision support system for anticipating shading change after tooth brightening by means applying multiple regression equations of CIELAB color coordinates. The difference between the original tooth color and the color difference expresses the color change after the whitening process. The color is defined by kappa value. The Decision support system predicted the color change using color metric values. A system for investigating the capital-constrained retailer's integrated purchase timing, quantity and decision on financing of seasonal products by Jinzhaoshi, et al., [16]. It gave the trade-off between information bonus and inevitable cost loss due to late-purchasing. The trade-off value decides an optimal purchase timing decision when it results low. It results as an intelligent decision support system for small retailers to take a decision at the corresponding time. Krishnan Krishnaiyer and F. Frank Chen [17] successfully implemented a Web-based Visual Decision Support System, which gave a method for achieving successful turnaround scheduling; capacity planning function and warehouse planning functions. This achieved a 100\% scheduled delivery rate, efficiency improved over the value stream and ongoing warning of client shipment to the client service agents. It was suitable for a high variety and low volume databases. Xiaochaung Yao, et al., [18] successfully integrated a decision Support System with Geographic Information System (GIS) for preventing and controlling locusts efficiently with accuracy and rapidly. It also provided the information about the tracking by means of Global Positioning System (GPS). Real-time data synchronization model and locust population estimation are developed and implemented to improve the decision-making of the system. An Intelligent decision system was designed for the milling systems by Vedika Agarwal, et al., [19] with a combination of fuzzy logic and Bayesian network. A model based leftover assessment approach is equipped for online fault detection and for finding the significant fault happening in the milling system. Fuzzy logic is utilized for remaining assessment for deciding the sort and extent of the fault happened, while the Bayesian network is utilized for investigating the underlying driver of the fault that has happened. Two contextual investigations are taken to show the viability of the approach.

A Game-theoretic approach was proposed to a traffic model by Nan $\mathrm{Li}$, et al., [20] for an autonomous driving. To overcome the challenge of time and effort of the decision and control algorithms to guarantee a sheltered and happy with driving. This model tests and compares various autonomous vehicles through their decision and control system and calibrates the attributes of an existing control framework through optimizing it. A Game-theoretic approach for the security and the challenges that arise in microcircuit industry due to the huge outsourcing of creation of Integrated Circuits, Protected innovation and Commercial OffThe-Shelf (COTS). An approach for avoiding or overcoming a major threat called as Hardware Trojan. This method tests digital circuits for decision making on the infected IC's by the intelligent attackers. A zero-sum game between the attackers and testers is done. The Nash equilibrium allows the detection of a possible attack was proposed by Charles A. Kamhoua, et al., [21]. An attacker and defender security game are proposed to identify and counter the malicious nodes in a Vehicular Ad-hoc Networks (VANETs) by Muhammad Mohsin Mehdi, et al., [22]. This was proposed mainly to facilitate road safety, transportation security, reliability, and management of nodes. A payoff matrix which determines the outcome of the specific game with the combination 
of action and reaction. Best strategies of attacker and defender are calculated by means of Nash equilibrium. This method performed better than the other game theory based approaches resulting in better throughput, retransmission attempts and different data drop rate for an attacker and defender. FaragAzzedin and Mohammed Yahaya proposed a Choking algorithm for BitTorrent using game theory [23]. Even the most successful protocol that is utilized for record sharing endures the issue of free riding. The proposed model both optimistic as the other un-checking algorithms. The game depends on the number of blocks downloaded or transferred from their adversaries. Fairness, robustness, and agility is calculated for the given model and proved that it is more efficient than other un-checking algorithms. Corine M. Laan [24] proposed an interdiction game that analyzes multiple simultaneous threats. Specifying the route of the intruders in a network differ from normal customer and intruders compete for the value of the network. A strategy that an intruder maximizes the throughput by selecting a fixed route, whereas an Interdictor minimizes it by selecting arrival rate at each node. The value and optimal strategies of the Interdictor remain same in the result of a game. Can Wang and Yu-qingTang [25] measure the system optimism and user equilibrium in a system through the result of Nash equilibrium?. Based on the fact that as the number of players grows, the result of Nash equilibrium leaves away from the system optimism, suggesting the inefficiency of management measures trying to achieve system optimism. This strategy called for a noncooperative game analysis. An iterated game theory is formulated through a structure for the examination of cyber switching assaults and control based relief in a cyber-empowered power framework by Abdallah Farraj [26]. The knowledge required here is only the indication of the neighborhood relative rotor speed. The game theory proposed here describes the connections of the gatherings and the impact of it on the dependability of the framework. Logical outcomes show the capability of the obliged controller for adjustment after some time utilizing zero determinant procedures. Feng zhou, et al., [27] gave a bi-level decision-making strategy in light of the Stackelberg game theory. A product portfolio is taken as the optimization problem, the product adoption maximization problem and product attributes are termed as the leader. Connection and coupling of these two advancement issues are tended to with a facilitate insightful enhancement methodology through enhanced greedy algorithm hybrid Taguchi genetic algorithm. The bi-level decision-making system for item configuration gave leeway over the existing viral promote strategies.

Ignacio Cepeda-Carrion, et al., [28] proposed the possible combinations by identifying and analysis between the various critical knowledge management processes. The superior value of the customer is calculated by means of the absorptive limit, knowledge exchange and knowledge application. A mix of three information administration process, fabricating a dynamic or higher request ability result in the superior value for clients. The drawback of the system is that it only considered few KM processes in effect on its customer value. Alexandre Barapet, et al., [29] designed an architecture for an ontology-driven knowledge management systems. To create a coherent web data layer for intranet learning and predictive analysis for vocabulary and semantics. It also defines vocabulary and semantics for knowledge sharing and re-usage projects in an organizational learning network. No mechanism provided for formal and informal processes is the main drawback of this System. Ming K. Lim, et al., [30] proposed a method for a sustainable supply chain management through the context of Knowledge management. This was done through a set of modeling methods to identify the driving and the dependence power of the management. The mentioned attributes are the most effective to enhance the performance of firms. Theoretical and managerial implications are also done to provide a better model. Gabriele Santoro, et al., [31] proposed a method for building a knowledge management system for IoT. The research employs an equation for structural modeling on a sample of 298 Italian firms from different sectors. Knowledge Management facilitates the open and collaborative system creation and the exploitation of internal and external flow of knowledge. The proposed system increased the innovation capacity of the system. Mian-Zhen Pan and Ji-Ye Mao [32] proposed a cross-boundary Knowledge Management system for the accomplishment of Enterprise Systems. Due to an extensive variety of authoritative units and stakeholder ES was implemented by alpha, which made cross-boundary difficult. To reveal the crossboundary mechanisms in KM by making a typical knowledge space, shared knowledge, and knowledge adoption. It developed the integrated model for both client and vendor side. It broadens the user participation in ES implementation context. By this way, it is evident that deploying game theoretical approach for decision-making in the online samples will result in efficient knowledge management and data aggregation. 
The different classifications of DSS and their relationships are discussed [33]. Fuzzy based DSS is proposed to validate the deferring accuracy based on intuitionistic fuzzy sets. Medical Intuitionistic Fuzzy Expert Decision Support System (MIFEDSS) helps in dragonising the data related to health care and decision-making is processed and recorded [34]. In the decision-making process, [35] discuss clinical applications and analyze the accuracy during the multiple data conflict.

In this paper, we propose a game theory based model to segregate the repeatedly occurring incident and we apply association rules over them so as to get a better confidence in taking the decision. The proposed model is combined with coding technique to make the transmission secure and reliable.

\section{Proposed research}

In general, Decision Support System (DSS), five components is required to fulfill the decisionmaking process to the users [36]. The components are computer information systems, Data management systems, knowledge management, Interface system and users.

The proposed Decision Making with Game Theory (DM-GT) system contains three major modules as shown in Fig. 1. It starts the process of collection of samples from various resources which act as an input. Secondly, a process of decisionmaking for analyzing the uncertainty in the game and the management module to manage the inference gained through the previous processes in the system. The system seems to be efficient by the process of effectively using the data that have been gained through previous processes.

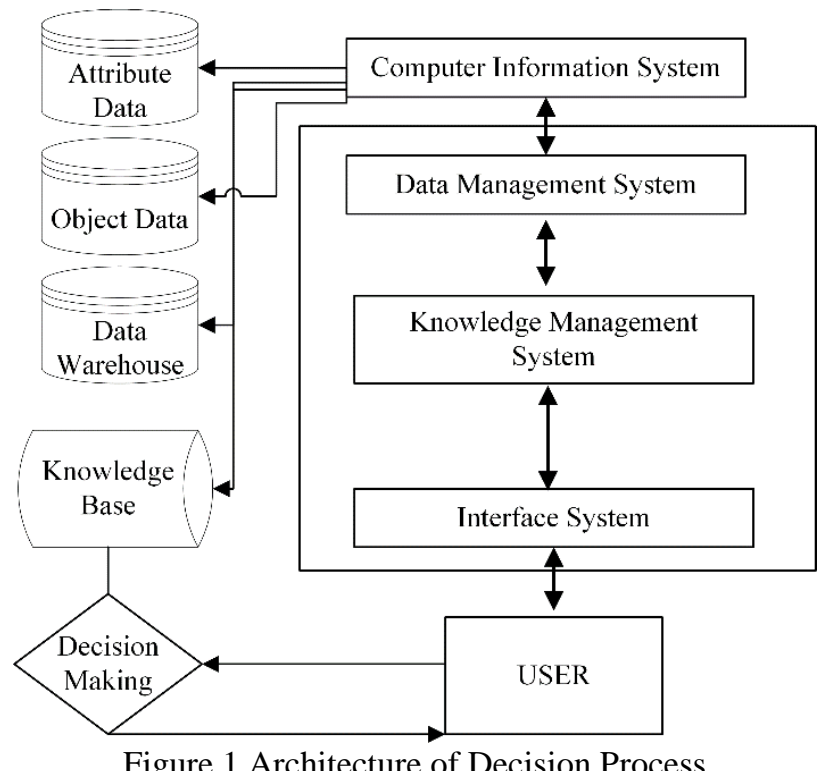

\subsection{Online samples}

The various datasets from E-Governance websites, social network site data, and patient data from the health care system are combined together. The combined data are statistically analyzed in both the ways of mean and variance statistics. The mean statistics are analyzed by implementing the same dataset in different areas and ensure that the data set is suitable for the implemented environments. The variance statistics are measured for calculating the stability of the data taken. Here, R tool is taken for the statistical analysis. The datasets of E-Commerce are taken from Google analytics, Dataset of Social network \& patient data is taken from the EU open data portal. Then the data sets are combined together to form a larger data set with related fields. Through the SPSS statistics in the $\mathrm{R}$ tool, combinations of three fields are given E-Governance, social network data, and patient data, then a common name is provided "Online Samples" and analysed by ANOVA test. Through the variance test, the significant difference is ensured. The data set is then exported online to a CSV file.

\subsection{Decision making}

The online sample data set that is created is enhanced to a game with two players to justify the model. It analyses various uncertainties, including performative uncertainty, analytic complexity, and narrative complexity. The performative uncertainty explains the various uncertainties that arise in the performance of the various factors such as playing platform, compiling performance, etc., then the uncertainty occurred are represented with the help of factor graphs. The variation in the graph shows the amount of uncertainty occurred in the implementation. The measured uncertainty ensures the performance of the proposed approach in the Fig. 2.

\subsection{Knowledge management}

As security plays an important role in the decision-making process as online samples are considered [37, 38]. We have proposed a new decision-making process by embedding secure coding technique to fulfill the user requirement. The new decision-making process is represented in Fig. 3.

The Knowledge management is processed by four elements they are, decision approval, Data mining, pattern aggregation and knowledge acquisition. 


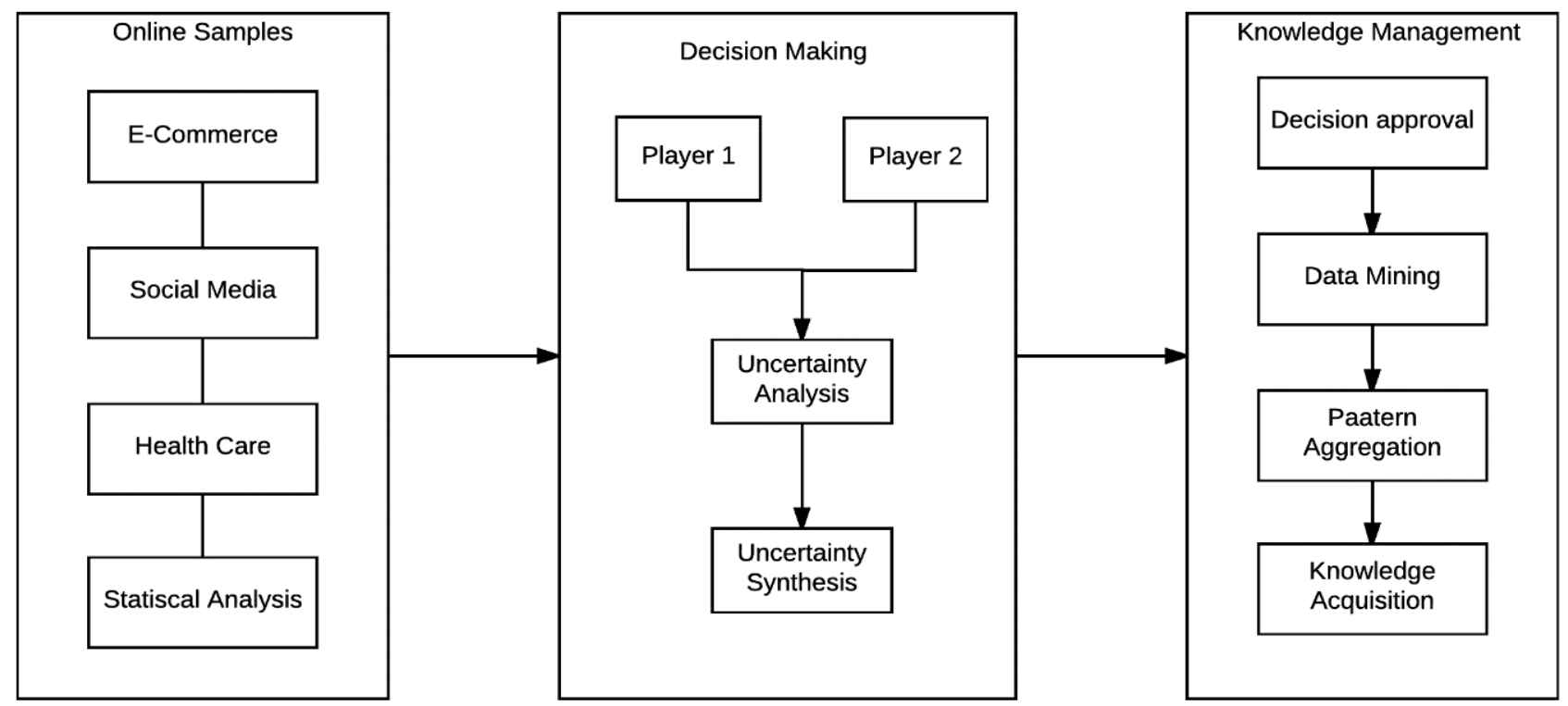

Figure.2 Knowledge acquisition process

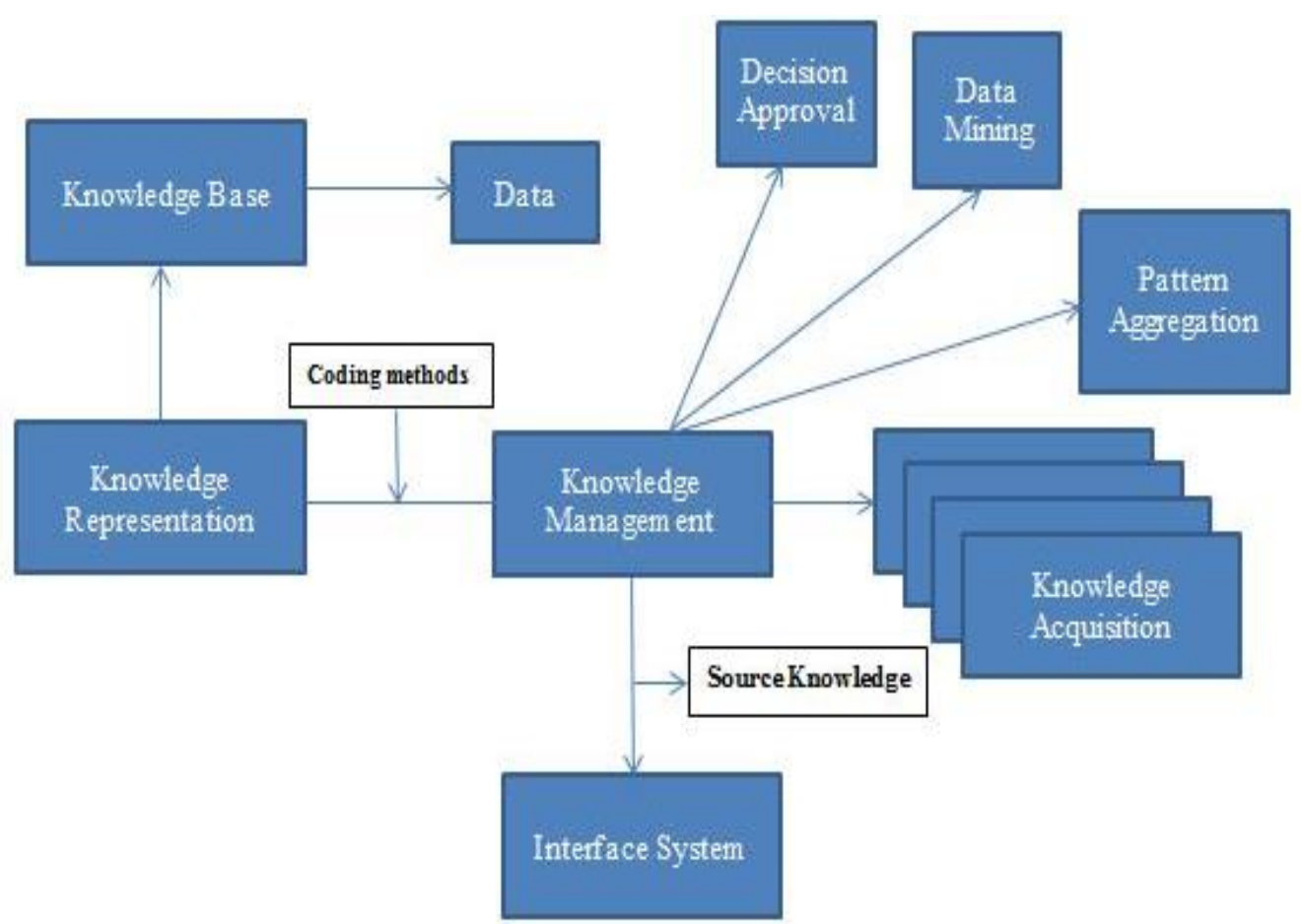

Figure.3 DM-GT for Knowledge acquisition

The data with least uncertainty is taken for storage. The data with the least uncertainty value is taken for decision-making. For decision-making, a Naïve Bayes classifier is used which is used to classify data sets that have huge variation [38].

The Bayesian model provides expected prediction as estimated based on the historical data in the context of multinomial distribution. The set of a procedure is taken to evaluate the prediction estimation for a set of events. The conditional probability is applied by posterior probability, likelihood, prior probability and normalization constant.
As considering conditional probability,

Posterior probability $\alpha$ Prior probability $\mathrm{X}$ Likelihood,

Where a posterior probability as

$\left.P\left(H \mid E_{1} E_{2} \ldots E_{n}\right)=\left[\begin{array}{llll}P\left(E_{1}\right. & E_{2} & \ldots & E_{n}\end{array}\right) \mid H\right] X P(H) /$ $P\left(E_{1} E_{2} \ldots E_{n}\right)$

Bayes theorem:

$P(R / K)=[(P(R) X P(K / R))] /(P(K))$ 


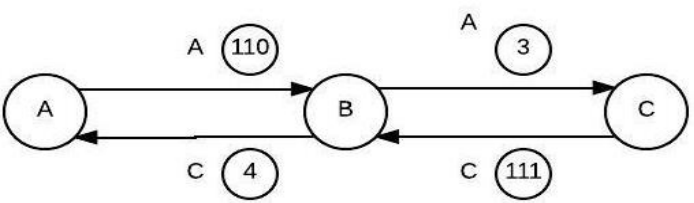

(a)

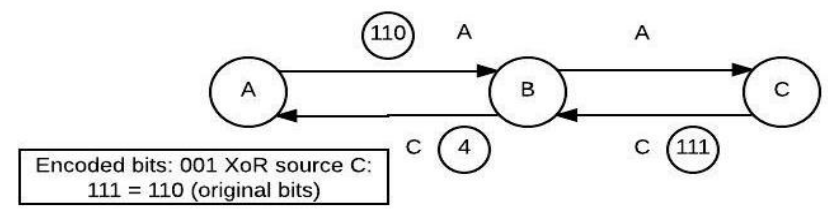

(c)

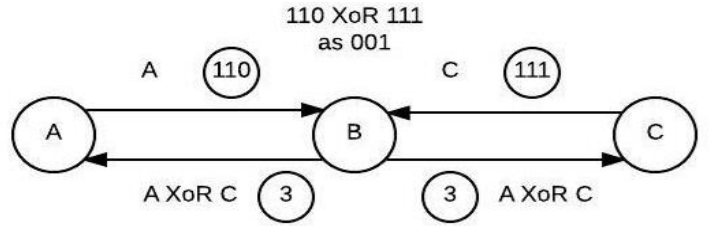

(b)

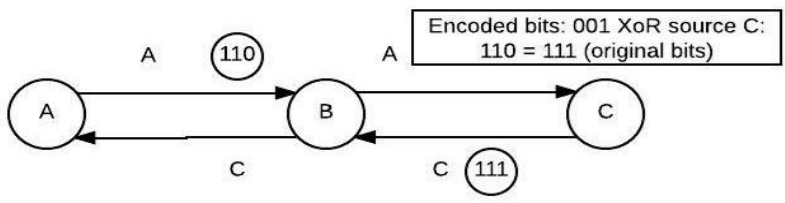

(d)

Figure.4 Coding approach process: (a) bit transferred A and C to B intermediate, (b) B compute XoR operation for both the data as encoded bits, (c) C as 111 bits and encoded bits 001 to form original bits as 110 (A), and (d) A as 110 bits and encoded bits 001 to form original bits as 111 (C)

Table 1. Performance of DM-GT

\begin{tabular}{|l|c|c|c|}
\hline \multicolumn{1}{|c|}{ Parameter } & Core-i3 & Core-i5 & Core-i7 \\
\hline Direct Data injection time & 0.823 & 0.795 & 0.652 \\
\hline Processed data injection time & 0.628 & 0.586 & 0.493 \\
\hline Data access time & 0.0856 & 0.0623 & 0.0521 \\
\hline Decision-Making time & 0.0325 & 0.0317 & 0.0298 \\
\hline Concurrent Access time & 0.036 & 0.031 & 0.029 \\
\hline
\end{tabular}

Where, $P(R \mid K)$ is a Posterior probability

$P(R)$ is a Prior probability

$P(K \mid R)$ is the likelihood

$P(K)$ is the priori probability

From Eq. (1), $\mathrm{K}$ can be classified based on the posterior probability $P(R \mid K)$.

$R^{*}=h_{R}(K)=\arg _{j=1 \ldots m^{\max } P\left(R_{j} / X\right)}$

With the bayes rule of ' $j$ ' attributes,

$P\left(R_{j} / K\right)=\left[\left(P\left(R_{j}\right) X P\left(K / R_{j}\right)\right)\right] /(P(K))$

Then the Bayesian classification rule Eq. (2),

$$
R^{*}=h_{\text {Bayes }}(K)=\arg _{j=1 \ldots m}{ }^{\max } P\left(K / R_{j}\right)
$$

When all attributes are conditionally independent,

$\left(R_{j} / K\right)=$ Integration from $i=1$ to $n P\left(K_{j}=K_{i} \mid R_{j}\right)$

Then the naïve Bayes classification rule,

$R^{*}=h_{N B}(K)=\arg _{j=1 \ldots m}{ }^{\max } P\left(R_{j}\right)$ Integration from

$i=1$ to $n P\left(K_{j}=K_{i} \mid R_{j}\right)$
Each value $X_{K}$ of attributes $X_{i}$ for each class $C_{j}$ with condition,

$P\left(K_{j}=K_{i} \mid R_{j}\right)=\left\{\begin{array}{c}N_{i j k} / N_{j}, X_{i} \text { is discrete } \\ g\left(X_{k}, \mu_{i j}, \sigma_{i j}\right) X_{i} \text { is Continues }\end{array}\right.$

$g(X, \mu, \sigma)=1 / \sqrt{2 \Pi \sigma} e^{(x-\mu) 2 / 2 \sigma 2}$

As naïve Bayes rule is formed as represented in Eq. (6).

Then, based on the classification values the probability of the frequently occurring data sets is founded. With the help of the frequently occurring datasets, a decision is taken. The data that have been involved in the decision-making. In this paper, we have proposed a coding technique during the knowledge extraction by the representation of data. In the coding technique, we have performed encoding and decoding process by performing arithmetic operations like exclusive OR. The arithmetic operation helps in obtaining the secure way of data transmission. The network coding data representation is represented in the figure. The coding technique is analyzed and the performance is measured in Fig. 4 (a), Fig. 4 (b), Fig. 4 (c), and Fig. 4 (d).

The performance of the data extraction is also measured. The behaviour of the players and the 
Table 2. Comparison of Evaluation of DM-GT

\begin{tabular}{|l|c|c|c|}
\hline \multicolumn{1}{|c|}{ Parameter } & $\begin{array}{c}\text { Traditional } \\
\text { Algorithms }\end{array}$ & $\begin{array}{c}\text { Data processing with } \\
\text { Fuzzy [19] }\end{array}$ & Proposed DM-GT \\
\hline Scalability & No & Yes & Yes \\
\hline Adaptability & No & Yes, with constraints & Yes, all time \\
\hline Data Security & No & Yes & Yes \\
\hline Concurrent Access & No & Yes, partially & Yes \\
\hline Data Storage time & 0.829 & 0.753 & 0.722 \\
\hline Data Access time & 0.652 & 0.568 & 0.419 \\
\hline Decision-making time & 0.735 & 0.694 & 0.485 \\
\hline Performance & Good & Better & Best \\
\hline
\end{tabular}

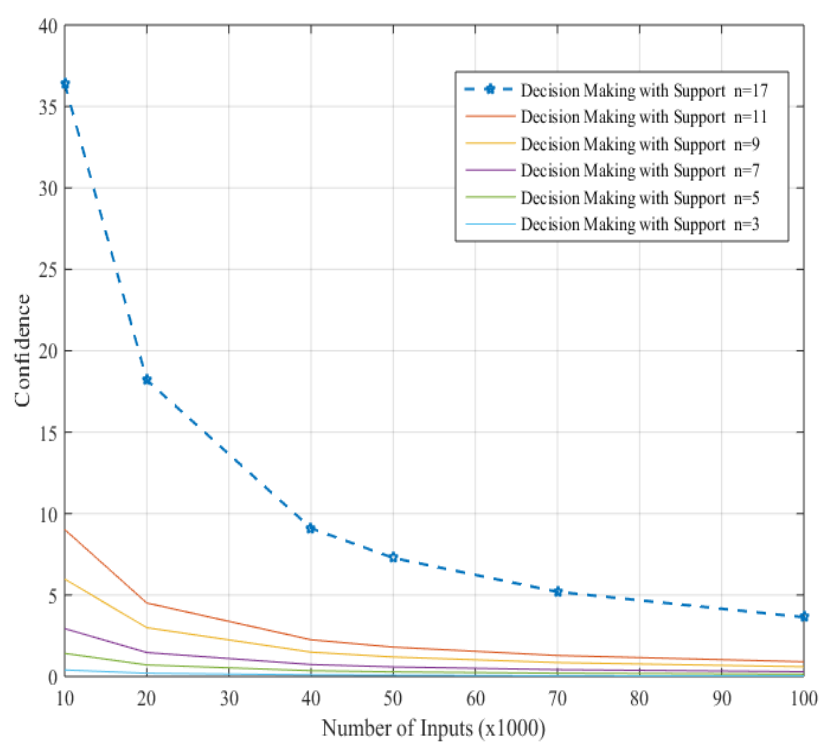

Figure.5 Scattering of different types of data over a region

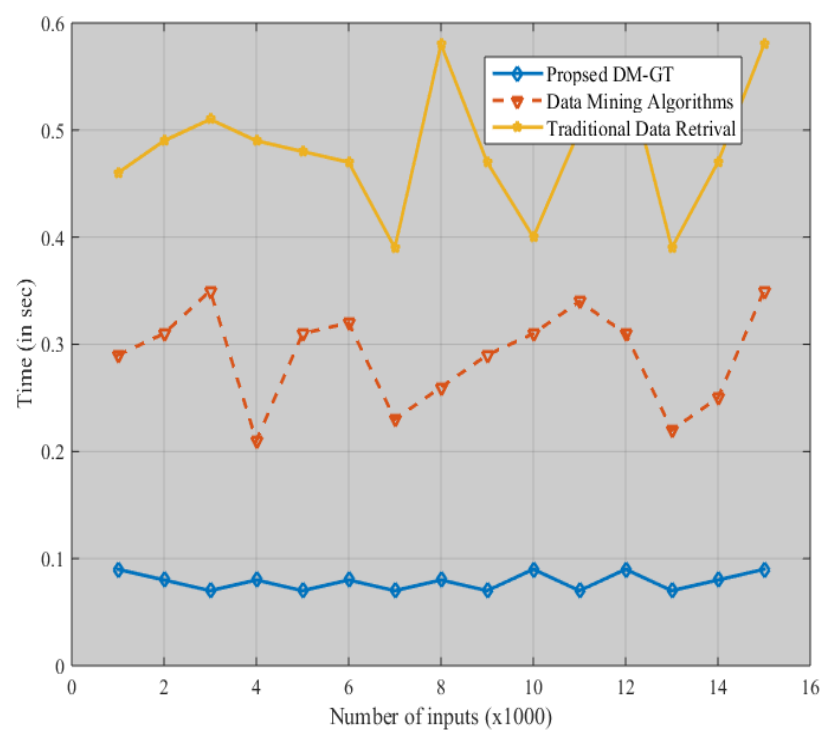

Figure. 6 Comparison of DM-GT with other data retrieval algorithms

system is observed by means of the pattern aggregation [39]. Statistics of the system show the pattern outcome of each play. The various patterns are then combined to give an analysis that the behaviour in a certain way. The certain method observed by pattern aggregation results each player behaviour analysis. The rules for the system are defined by means of the acquisition process. Thus, by acquiring the domain knowledge, scaling up the process of this expert system is done. This is based on the reuse-based approach which standardizes and shared across a broad community easily. This is developed using Web Ontology Language (OWL) [40].

\section{Performance analysis}

The performance of the proposed algorithm is analyzed in various simulation environments. Table 1 shows the performance of the DM-GT algorithm in different platforms and different specifications. Fig. 5 shows the scattering of various types of data over a particular region. Through Fig. 5, we infer that E-Commerce data is contributing an equal portion with respect to social data. Also, the contribution of the social data and E-Commerce data dominates the region.

\subsection{Data analysis with respect to the proposed algorithm}

A comparison of our proposed DM-GT algorithm with traditional data retrieval mechanism is plotted in Fig. 6. Comparison of the proposed system with various parameters involved in data management is presented in Table 2 .

Decision-making process softens the curve by iteratively doing the same function. To increase the maximization function, we make the process several times with increased support as shown in Fig. 7. It shows that if we increase the number of iteration and support, then confidence has proportionally increased.

\subsection{Quality analysis}

The quality can be analyzed by several parameters, namely throughput, delay, etc. Here, we have compared our proposed DM-GT with other existing algorithms like MEWS [42] and CDSS [43]. 


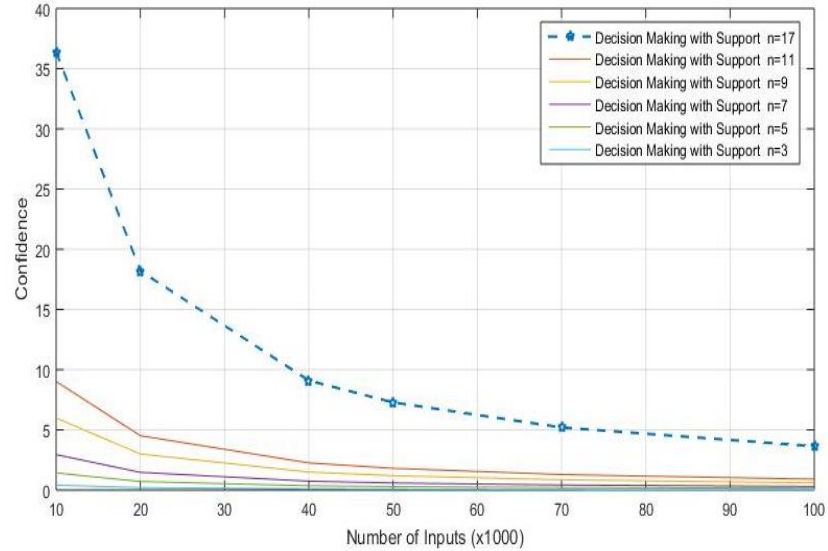

Figure.7 Decision-making process by iteration

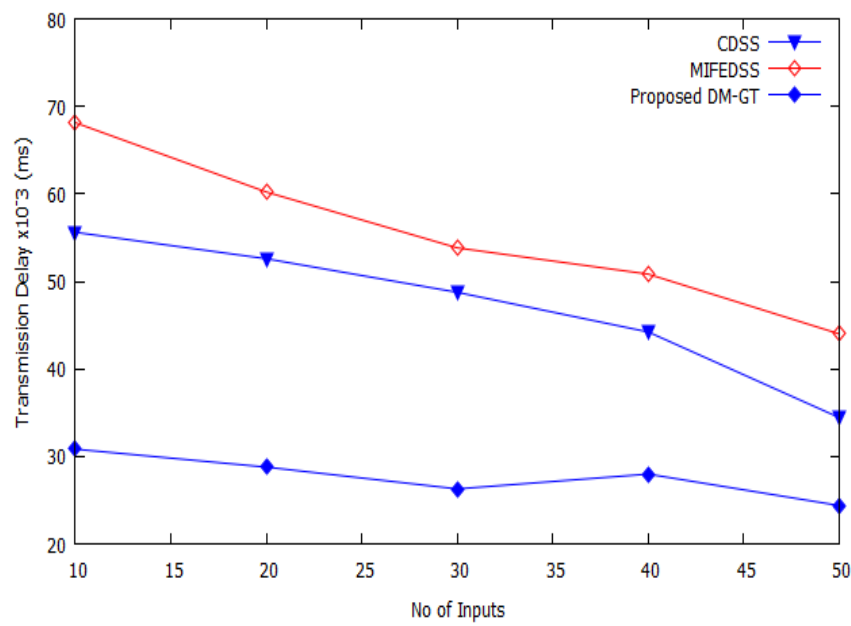

Figure.8 Transmission delay analysis

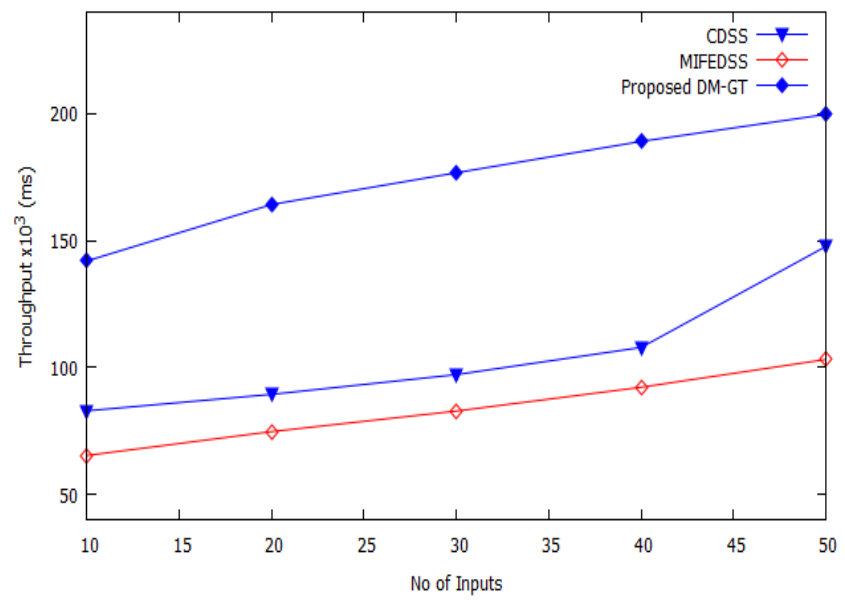

Figure.9 Throughput Analysis

The transmission delay is calculated by varying the number of inputs (Data) as shown in Fig. 8. It is inferred that the transmission delay gets decreased for the proposed DM-GT than the existing algorithms such as MEWS [34], and CDSS [35].

The throughput is calculated by varying the number of inputs (Data) as shown in Fig. 9. It is inferred that the throughput gets increased for the proposed DM-GT than the existing algorithms such as MEWS [34], and CDSS [35].

\section{Conclusion}

Data management is difficult when there is unstructured data. In this paper, we formulated an algorithm that simulates a game using the E-Commerce inputs and the decision-making is carried out with the help of game theoretical approach. We have combined knowledge data input with coding technique to make the proposed algorithm more secure and reliable. Traditional data management algorithms consume more time and complex mechanisms to take a decision. Thus, our proposed DM-GT outperforms the traditional data retrieval algorithms under our considered scenario. Also, performance analysis shows that our algorithm is an efficient, quality in terms of transmission delay and throughput. The future scope of this paper is planned to implement the same using fuzzy improved inputs and data, refining is planned to be deployed in the uncertain situation.

\section{References}

[1] D. McDuff and R. Kaliouby, "Applications of Automated Facial Coding in Media Measurement", IEEE Transactions on Affective Computing, Vol.8, No.2, pp.48-160, 2017.

[2] R. Grzeszick, A. Plinge, and G. A. Fink, "Bagof-Features Methods for Acoustic Event Detection and Classification", IEEE/ACM Transactions on Audio, Speech, And Language Processing, Vol.25, No.6, pp.1242-1252, 2017.

[3] H. Lin, JiaJia, J. Qiu, Y. Zhang, Guangyao Shen, L. Xie, J. Tang, L. Feng, and T. Seng Chua, "Detecting Stress Based on Social Interactions in Social Networks", IEEE Transactions on Knowledge And Data Engineering, Vol.29, No.9, pp.1820-1834, 2017.

[4] L. Yu, H. Chen, Q. Dou, J. Qin, and P. Ann Heng, "Integrating Online and Offline ThreeDimensional Deep Learning for Automated Polyp Detection in Colonoscopy Videos", IEEE Journal Of Biomedical And Health Informatics, Vol.21, No.1, pp.65-75, 2017.

[5] T. Zhang, J. H. D. Cho, and C. Zhai, "Understanding User Intents in Online Health Forums", IEEE Journal of Biomedical And Health Informatics, Vol.19, No.4, pp.1392-1398, 2015.

[6] M. H. Mahmood, Y. Díez, J. Salvi, and X. Lladó, "A collection of challenging motion segmentation benchmark datasets", Elsevier, Pattern Recognition, Vol.61, pp.1-14, 2017. 
[7] C. Grajeda, F. Breitinger, and I. Baggili, "Availability of datasets for digital forensics and what is missing", Elsevier, Digital Investigation, Vol.22, pp.S94-S105, 2017.

[8] H. Caple and J. S. Knox, "Genre(less) and purpose(less): Online news galleries", Elsevier, Discourse, Context \& Media, University of New South Wales, Sydney, Australia, Accepted for publication, 2017.

[9] J. Zhang, W. Li, P.O. Ogunbona, P. Wang, and C. Tang, "RGB-D-based action recognition datasets: A survey", Elsevier, Pattern Recognition, Vol.60, pp.86-105, 2016.

[10] H. Jalota, M. Thakur, and G. Mittal, "A Credibilistic Decision Support System for Portfolio Optimization", Elsevier, Applied Soft Computing, Vol.59, Iss. C, pp.512-528, 2017.

[11] H. Chi, Z. Lin, H. Jin, B. Xu, and M. Qi, “A decision support system for detecting serial crimes", ACM Knowledge-Based Systems, Vol.123, Iss. C, pp.88-101, 2017.

[12] A.R. Xambre, L. Teixeira, and P.Vilarinho, "A Decision Support System for the design of cellular Manufacturing Systems: database conceptualization", In: Proc. of Computer Science, Vol.100, pp.164-170, 2016.

[13] G. Yang, L. Liu, P. Guo, and M. Li, “A flexible decision support system for irrigation scheduling in an irrigation district in China", Agricultural water management, Vol.179, pp.378-389, 2016.

[14] G. Legien, B. Sniezynski, D. WilkKołodziejczyk, S. Kluska-Nawarecka, E. Nawarecki, and K. Jaśkowiec, "Agent-based Decision Support System for Technology Recommendation", In: Proc. of Computer Science, Vol.108, pp.897-906, 2017.

[15] B. Thanathornwong, S. Suebnukarn, and K. Ouivirach, "Decision support system for predicting color change after tooth whitening", Computer Methods and Programs in Biomedicine, Vol.125, pp.88-93, 2016.

[16] J. Shi, J.E. Guo, and R.Y. Fung, "Decision support system for purchasing management of seasonal products: A capital-constrained retailer perspective", Expert Systems with Applications, Vol.80, pp.171-182, 2017.

[17] K. Krishnaiyer and F.F. Chen, "Web-based Visual Decision Support System (WVDSS) for letter shop", Robotics and Computer-Integrated Manufacturing, Vol.43, pp.148-154, 2017.

[18] X. Yao, D. Zhu, W. Yun, F. Peng, and L. Li, "A WebGIS-based decision support system for locust prevention and control in
China", Computers and Electronics in Agriculture, Vol.140, pp.148-158, 2017.

[19] B.K. Panigrahi, and P.M.V. Subbarao, "Intelligent Decision Support System for Detection and Root Cause Analysis of Faults in Coal Mills", IEEE Transactions on Fuzzy Systems, Vol.25, No.4, pp.934-944, 2016.

[20] N. Li, D.W. Oyler, M. Zhang, Y. Yildiz, I. Kolmanovsky, and A.R. Girard, "Game Theoretic Modeling of Driver and Vehicle Interactions for Verification and Validation of Autonomous Vehicle Control Systems", IEEE Transactions on Control Systems Technology, Vol. PP, Iss. 99, pp.1-16, 2017.

[21] C.A. Kamhoua, H. Zhao, M. Rodriguez, and K.A. Kwiat, "A game-theoretic approach for testing for hardware Trojans", IEEE Transactions on Multi-Scale Computing Systems, Vol.2, No.3, pp.199-210, 2016.

[22] M.M. Mehdi, I. Raza, and S.A. Hussain, "A game theory based trust model for Vehicular Ad hoc Networks (VANETs)", Computer Networks, Vol.121, pp.152-172, 2017.

[23] F. Azzedin and M. Yahaya, "Modeling BitTorrent choking algorithm using game theory", Future Generation Computer Systems, Vol.55, pp.255-265, 2016.

[24] C.M. Laan, T. van der Mijden, A.I. Barros, R.J. Boucherie, and H. Monsuur, "An interdiction game on a queueing network with multiple intruders", European Journal of Operational Research, Vol.260, No.3, pp.1069-1080, 2017.

[25] C. Wang, and Y.Q. Tang, "The discussion of system optimism and user equilibrium in traffic assignment with the perspective of game theory", Transportation Research Procedia, Vol.25, pp.2974-2983, 2017.

[26] A. Farraj, E. Hammad, A. Al Daoud, and D. Kundur, "A game-theoretic analysis of cyber switching attacks and mitigation in smart grid systems", IEEE Transactions on Smart Grid, Vol.7, No.4, pp.1846-1855, 2016.

[27] F. Zhou, R.J. Jiao, and B. Lei, "Bi level GameTheoretic Optimization for Product Adoption Maximization Incorporating Social Network Effects", IEEE Transactions on Systems, Man, and Cybernetics: Systems, Vol.46, No.8, pp.1047-1060, 2016.

[28] I. Cepeda-Carrión, S. Martelo-Landroguez, A. Luis Rodríguez, and A. Leal Millán, "Critical processes of knowledge management: An approach toward the creation of customer value", European Research on Management and Business Economics", Vol.23, pp.1-7, 2017. 
[29] A. Barão, J.B. de Vasconcelos, Á. Rocha, and R. Pereira, "A knowledge management approach to capture organizational learning networks", International Journal of Information Management, Vol.37, No.6, pp.735-740, 2017.

[30] M.K. Lim, M.L. Tseng, K.H. Tan, and T.D. Bui, "Knowledge management in sustainable supply chain management: improving performance through an interpretive structural modelling approach", Journal of Cleaner Production, Vol.162, pp.806-816, 2017.

[31] G. Santoro, D. Vrontis, A. Thrassou, and L. Dezi, "The Internet of Things: Building a knowledge management system for open innovation and knowledge management capacity", Technological Forecasting and Social Change, In Press, pp.1-8, 2017.

[32] M.Z. Pan and J.Y. Mao, "Cross Boundary Mechanisms for Knowledge Management by User Representatives in Enterprise Systems Implementation", IEEE Transactions on Engineering Management, Vol.63, No.4, pp.438-450, 2016.

[33] E.V. Ocalir-Akunal, "Decision Support Systems in Transport Planning", Procedia Engineering, Elsevier Publications, Vol.161, pp.1119-1126, 2016.

[34] H. Jemal, Z. Kechaou, and M. B. Ayed, "Enhanced Decision Support Systems in Intensive Care Unit Based on Intuitionistic Fuzzy Sets", Journal on Advances in Fuzzy Sets, Hindawi, https://doi.org/10.1155/2017/7371634, 2017.

[35] A. Fan, D. Lin, and Y. Tang, "Clinical Decision Support Systems for Comorbidity: Architecture, Algorithms, and Applications", In: Proc. of International Journal of Telemedicine and Applications, https://doi.org/10.1155/2017/156 2919, 2017.

[36] D. J. Power, and R. Sharda, "Decision Support Systems", Springer Handbook of Automation, pp.1539 - 1548, 2009.

[37] S. H. El-Sappagh and S. El-Masri, "A distributed clinical decision support system architecture", Journal of King Saud University Computer and Information Sciences, Elsevier Publications, Vol.26, No.1, pp.69-78, 2014.

[38] K.M.R. Malini and R. Lakshmi, "A secure Decision Making Process in Health Care System Using Naïve Bayes Classifier", In: Proc. of International Journal for Trends in Engineering \& Technology, Vol.4, No.1, pp.4649, 2015.

[39] H. A. Simon, "Rational Decision Making in Business Organizations", The American
Economic Review, Vol.69, No.4, pp.493-513, 1979.

[40] H.A. Salah, "Ontology development (OWL\&UML) methodology of web-based Decision Support System for water management", In: Proc. of the 6th International Conference on Electronics, Computers and Artificial Intelligence, Doi: 10.1109/ ECAI.2014.7090217 2014. 